\title{
REGULACIONES NACIONALES SOBRE EL CURRÍCULUM: ARGENTINA, LAS NUEVAS FORMAS DE GOBIERNO E INSTRUMENTOS CURRICULARES
}

\author{
Silvina Feeney* \\ Universidad Nacional de General Sarmiento (UNGS), Buenos Aires, Argentina \\ Daniel Feldman* \\ Universidad de Buenos Aires (UBA), Buenos Aires, Argentina
}

RESUMEN: Desde los años '80, los sistemas educativos nacionales se han visto inmersos en profundos movimientos de cambio en su gobierno desde o bacia el centro. Estas opciones fueron respuestas localizadas a cambiantes relaciones entre políticas, formas de gobierno y construcción de legitimidad y han puesto en foco el debate acerca del control sobre la escolaridad y el currículum. Este trabajo toma como eje la trayectoria de Argentina, situándola en el marco de distintas experiencias de países de América del Sur, para analizar qué se decidió sobre el currículum en las últimas décadas. Su propósito es alimentar una reflexión sobre el problema y los debates en torno a las regulaciones nacionales sobre el currículum.

Palabras clave: Políticas curriculares. Gobierno del currículum. Instrumentos curriculares.

\section{NATIONAL REGULATIONS ON THE CURRICULUM: ARGENTINA, THE NEW GOVERNANCE FORMATS AND CURRICULAR TOOLS}

ABSTRACT: Since the 80's, national education systems have been immersed in deep change movements in its government from or towards the center. These options were localized responses to changing relationships between policies, governance and building legitimacy. They put into focus the debate over school's control and its curriculum. This work analyzes what was decided about the curriculum in recent decades, taking as axis the path of Argentina and placing it in the context of different experiences in other countries in South America. Its purpose is to nourish a reflection on the problems and debates on national regulations on the curriculum.

Keywords: Curriculum policies. Curriculum governance. Curricular tools.

http://dx.doi.org/10.1590/0102-4698153047

* Magíster en Didáctica, Universidad de Buenos Aires-Universidad Nacional de General Sarmiento. Argentina. Correo electrónico: < silvinafeeney@gmail.com>.

" Licenciado en Ciencias de la Educación, Universidad de Buenos Aires, Argentina. Correo electrónico: $<$ danielf51@yahoo.com.ar $>$. 


\section{REGULACT̃ES NACIONAIS SOBRE O CURRÍCULO: ARGENTINA, AS NOVAS FORMAS DE GOVERNO E INSTRUMENTOS CURRICULARES}

RESUM0: Desde os anos 1980, os sistemas educativos nacionais se viram imersos em profundos movimentos de mudança em seu governo desde ou em direção ao centro. Essas opções foram respostas localizadas às mudanças nas relações entre políticas, formas de governo e construção de legitimidade. Elas colocaram em foco o debate sobre o controle da escolaridade e do currículo. Este trabalho toma como eixo a trajetória da Argentina, situando-a no marco de distintas experiências de países da América do Sul, ao analisar o que se decidiu sobre o currículo escolar nas últimas décadas. Seu propósito é alimentar uma reflexão sobre o problema e os debates em torno das regulações nacionais sobre o currículo.

Palavras-chave: Políticas curriculares. Governo do currículo. Instrumentos curriculares.

\section{PRESENTACIÓN}

Desde los años'80 en adelante, los sistemas educativos nacionales se han visto inmersos en profundos movimientos de cambio en su gobierno desde o hacia el centro, poniendo en foco el debate sobre cómo se ejerce el control sobre la escolaridad, en general, y sobre el currículum en particular. Actualmente, la discusión sobre el punto es intensa, tanto en los aspectos referidos a las posibilidades y legitimidad de distintas alternativas para reformular las relaciones de autoridad y toma de decisiones sobre el currículum; como a la regulación sobre el contenido escolar que representan distintos instrumentos (marcos, estándares, parámetros, etc.) en el tránsito hacia formas de autoridad más distribuidas y a la de asegurar mayores estándares de calidad y equidad.

Este trabajo propone un breve análisis de la trayectoria de un país con tradición centralista como Argentina -que, durante veinte años de reforma continuada y caracterizada por importantes vaivenes, expresó cambios importantes en estas dos dimensiones de las políticas curriculares- situándolo en el marco de distintas experiencias sudamericanas. De antemano es necesario aclarar que no se trató de realizar un estudio comparado de políticas o alguna periodización de estrategias según "generación de reformas", sino solo de relevar alternativas que se configuraron durante el período para alimentar una reflexión en torno a las regulaciones nacionales sobre el currículum. El propósito principal del texto es la reconstrucción de un caso nacional de políticas relativas a las normas curriculares y 
la sugerencia de un marco interpretativo para su estudio. Su punto de vista sobre el currículum es específico y, por consiguiente, limitado.

Independientemente de los variados alcances que se atribuyen al concepto (PINAR et al, 1995; PINAR, 1998; DE ALBA, 1995; ORNSTEIN; BEHAR, 1995; DOLL, 1997), el currículum es siempre una construcción histórica y cultural cuyas formas se moldean de acuerdo con las distintas configuraciones sociales que lo contienen. En este trabajo se tratan los problemas que se conjugan en torno a la definición del currículum oficial -el que emana como una regulación del estado- cuyos significados y características dependen de la forma en que se construyen las tradiciones políticoeducativas (PALAMIDESSI; FELDMAN, 2003). En este marco, las configuraciones y dinámicas que adopta el currículum son producto de las diversas maneras en que se relacionan el campo educativo, el Estado y las organizaciones civiles y de cómo se concretan proyectos sociales e intenciones político-pedagógicas en la vida de las instituciones educativas (COX, 1989). Por eso, estudiar el currículum -y los instrumentos utilizados para su gestión y cambio- es estudiar la trayectoria de formas institucionalizadas de intervención educativa, caracterizadas por estrategias de clasificación, control y gobierno propias de cada país (GOODSON, 2006). Es así que el currículum, en tanto pauta normativa oficial, queda simultáneamente vinculado con los procesos de construcción del Estado y con la regulación de prácticas a nivel local (VAN DEN AKKER, 2004). En la articulación de estos procesos y prácticas emergen las políticas mediante las que se pretendió ordenar la transmisión escolar en el último período.

\section{AÑOS DE REFORMAS SOBRE LA EDUCACIÓN Y EL CURRÍCULUM}

Distintos países de la región se involucraron en importantes reformas educativas desde la década de 1990. Estas incluyeron modificaciones en la estructura de niveles, extensión de la obligatoriedad escolar, procesos de descentralización que afectaron el financiamiento, la toma de decisiones, la administración y la gestión de los sistemas (GAJARDO, 1999; TEDESCO; TENTI FANFANI, 2001; KAUFMAN; NELSON, 2005; KRAWCZYK; VIEIRA, 2009; AGUERRONDO; NÚÑEZ PRIETO; WEINSTEIN, 2010). Es algo todavía en debate la apreciación de los efectos de estas reformas y de su relación con las formas dominantes de control y distribución de bienes en nuestras sociedades. Pero está claro que configuraron un nuevo escenario que motorizó variadas acciones sobre el currículum 
y puso en cuestión sus variables básicas. Fueron modificados niveles y ciclos escolares, redefinidas formas de gobierno escolar y distribuidas responsabilidades sobre la definición del contenido escolar y la enseñanza, se amplió la obligatoriedad escolar, se desarrollaron nuevas formas de regulación y se generalizaron los dispositivos de evaluación. Como señala Palamidessi (2006, p. 2)

En este contexto, todos los países de la región atravesaron procesos amplios y sistemáticos de revisión y renovación de sus definiciones curriculares [...] Se trató de uno de los movimientos más ambiciosos y comprensivos de revisión de los contenidos escolares desde la instauración de los sistemas educativos hacia fines del siglo XIX y comienzos del siglo XX.

Estos procesos de reforma sobre la escolaridad estuvieron articulados con la re-construcción de las democracias en muchos países de América del Sur y con procesos de reestructuración de las economías y de las sociedades cuyo signo varió de manera muy importante según etapas y países durante este período.

Estas políticas formaron parte de un proceso general de expansión de la educación a nivel mundial que se caracterizó por la instalación progresiva y relativamente firme de la educación primaria y por movimientos mucho más escalonados y lentos de incorporación de mayor cantidad de estudiantes, provenientes de distintos sectores sociales, a la educación media, tradicionalmente de carácter preparatorio ${ }^{1}$. La progresiva apertura de la educación media sufrió un cambio cualitativo a partir de sucesivas ampliaciones del tramo de escolaridad obligatoria más allá del tradicional nivel primario, cuya duración oscilaba entre seis y ocho años. Por ejemplo, Uruguay creó un Ciclo Básico de educación secundaria obligatorio a fines de los años de 1970 y extendió en 2008 la obligatoriedad del Ciclo Superior; Argentina impuso en los años 90 la obligatoriedad de 10 años de educación y la amplió en 2006 al nivel secundario completo; en Brasil, la obligatoriedad de educación básica (Enseñanza Fundamental y Enseñanza Media) se vio consolidada en 2009 y ampliada al período entre 4 y 17 años de edad en 2013; Chile amplió la obligatoriedad de la enseñanza básica de 8 años a la enseñanza media de 4 años y Colombia tiene una educación básica y obligatoria de 9 años que comprende la educación primaria y la secundaria básica. Estas ampliaciones en la obligatoriedad supusieron un creciente esfuerzo por resolver problemas de mantenimiento, avance y graduación de los estudiantes, cualitativamente superiores a los que proponía la extensión de la cobertura, problema característico de períodos anteriores. También 
conllevaron, de hecho, una redefinición de las funciones de los distintos niveles y ciclos que componen cada sistema escolar: implicaron un cambio de profundas proporciones en las grandes divisiones históricas de las funciones escolares, que acompañó una tendencia mundial respecto de la educación secundaria: "[...] esta se fue distanciando de su estricto rol de preparatoria para estudios superiores $[. .$.$] y se fue$ acercando en forma paulatina a una función educativa de carácter más general [...] Más que preuniversitaria, esta educación secundaria es hoy post-primaria [...]" (BELLEI CARVACHO, 2012, p. 215).

El conjunto de exigencias, asociado a un generalizado aumento de la escolaridad y de la obligatoriedad escolar se realizó en contextos no siempre favorables. Como señalaban Tedesco y López (2002, p. 61): "[...] En síntesis, América Latina debe enfrentar -al mismo tiempo- la diversidad y la desigualdad y debe hacerlo en un contexto en el cual las tendencias estructurales generan fuerte incertidumbre y riesgos de mayores niveles de exclusión social". Fue necesario, entonces, enfrentar el desafío que representaron, para las promovidas estrategias de expansión de la educación, los fenómenos de exclusión, desgranamiento y abandono de la escolaridad por parte de una importante cantidad de alumnos. De allí la importancia que adquirieron distintos programas que procuraron atender los problemas de mantenimiento de la escolaridad y mejora de los aprendizajes de alumnos de sectores desfavorecidos (FELDMAN, ATORRESI, MEKLER, 2013).

En términos generales, varias líneas de discursos convergieron - de manera no necesariamente unificada y, en muchos casos, representando intereses diferenciados, sostenidos políticamente por distintas fuerzas- para sostener la necesidad y pertinencia de las reformas educativas de estas décadas. Una de ellas estaba relacionada, como señalan Kaufman y Nelson (2005), con la importancia de la educación para el desarrollo y la modernización, asociada con un creciente interés a nivel internacional y local por mejorar el rendimiento de los estudiantes. Esta línea argumental estuvo respaldada y profusamente financiada por los organismos multilaterales como el Banco Mundial y el BID y mostraba la convicción de la importancia de invertir en capital humano para aumentar la competitividad en mercados globalizados. Otro conjunto de argumentos estaba relacionado con el afianzamiento y fortalecimiento de la democracia, en muchos países de la región reestablecida tras décadas de gobiernos militares $\mathrm{y}$ frecuentes interrupciones de la vida institucional y las normas constitucionales. La creación de democracias más sólidas, marcadas por crecientes grados de participación ciudadana, se ligó de manera 
profunda con la ampliación de derechos y la necesidad de ofrecer mayores oportunidades de acceso a bienes sociales como la educación, la salud y la vivienda, además de mejoras sustantivas en el trabajo.

Distintos factores confluyeron para articular las fuerzas que se conjugaron en torno a estas reformas. Durante los últimos 30 años mejoraron los indicadores, pero sin embargo se mantuvieron altos grados de desigualdad educativa. Aumentó el número de niños y jóvenes que asisten a la escuela y se amplía la cobertura, pero los sectores más integrados a la vida económica y los que habitan en zonas urbanas encontraron mejores oportunidades. Tampoco los ritmos de avance de muchos niños y jóvenes latinoamericanos eran los esperados para sus edades, lo que se manifestó en la repitencia, la sobre-edad y el abandono. Según datos del SERCE (2008) en el promedio de doce países de la región había una diferencia de más de 30 puntos porcentuales entre el ingreso y la terminación oportuna del nivel o ciclo. Estas diferencias se profundizaban cuando se toman en consideración las zonas rurales y la inserción laboral de las familias de los estudiantes. Como señalan Cervini y Tenti Fanfani (2007), los países habían logrado mantener un grado de expansión cuantitativa relevante, pero esa expansión coexistía con importantes desigualdades en los recorridos, en la exitosa terminación de los estudios y en los niveles de aprendizaje. Estas constataciones motivaron la búsqueda de alternativas para lograr mayor equidad. Además, las mediciones de resultados de aprendizaje en distintos instrumentos internacionales ubicaban en posiciones rezagadas a los países de la región participantes, lo cual generó inquietud en autoridades educativas y ciertos sectores políticos y de la opinión pública. De hecho, ha crecido la preocupación en aquellos países que partiendo de mejores resultados han retrocedido en sus posiciones, como los casos de Uruguay y Argentina. También gran parte de la crítica que surgió en los años ochenta y noventa estuvo dirigida, con las lógicas diferencias debidas a las distintas estructuras nacionales, hacia la excesiva centralización y rigidez organizacional en la gestión de la educación. De allí que se promovieron políticas de modernización, rendición de cuentas, mayor preocupación por la eficacia y la competitividad, junto con un papel más relevante de la evaluación sistemática.

Puede decirse que la reconstitución de las sociedades latinoamericanas, la nueva ola de financiamiento con sus correlatos de "reformas estructurales", el agotamiento del modelo de crecimiento educativo exclusivamente por cobertura, la preocupación por el aprendizaje, la terminalidad y los requerimientos de un aumento 
importante y extendido de la obligatoriedad escolar, fueron factores que se conjugaron para promover la reorganización de los sistemas escolares en mucho países de la región, sumado a la sanción de leyes educativas y un intenso trabajo sobre el contenido de la escolaridad a través del currículum y de la evaluación.

\section{DISTRIBUCIÓN DE AUTORIDADE INSTRUMENTOSPARA LA REGULACIÓN DEL CURRÍCULUM}

Las reformas educativas de las últimas dos décadas en América del Sur se llevaron adelante en un escenario de mayor complejidad social marcado por una modificación a nivel internacional del papel de los estados nacionales en el gobierno de los asuntos educativos. Por ejemplo, si se toma el eje "centralización-descentralización" de las decisiones, puede notarse que distintos países han producido reformas en sentido inverso (KOGAN, 1996; KUIPER; AKKER, 2005). El rol del estado en educación parece estar puesto en cuestión y los mecanismos de gobierno y de gestión de distintos sistemas nacionales transitan hacia formatos diferentes de los que se utilizaron durante el período de su constitución y asentamiento. El conjunto de políticas sobre el currículum del período muestra, en resumen, un cambio de importancia en las formas de gobernar el contenido que trasciende las coyunturas de reforma y probablemente implica para la región un cambio cualitativo es sus maneras de tratar la regulación del currículum.

Durante las últimas décadas, los problemas relativos a la descentralización o centralización de responsabilidades y misiones desde el estado nacional hacia entidades subnacionales (Departamentos, Provincias, Estados) o municipios fue un aspecto principal de las reformas educativas en la región, lo que incluyó las responsabilidades y capacidades de decisión sobre el currículum. Estos procesos tuvieron distintos puntos de partida, debido a que las estructuras históricas para el gobierno educativo en cada país generaron distintas trayectorias. En ese sentido, todo cuadro general muestra un agregado que tiende a homogeneizar realidades diversas. Pero, en verdad, las tendencias descriptas en el apartado anterior se expresaron de manera diferenciada en distintos países y el cuadro de ninguna manera es homogéneo. En buena medida, porque las fuerzas de cambio operaron sobre distintas tradiciones, distintas formas de gobierno, distintos contextos políticos y distintas situaciones de cada sistema educativo en cuanto a extensión, cobertura y logros. Sin embargo, pese a estas diferencias, por un lado se verificó un creciente cambio en las formas de gobierno de los sistemas escolares, rebalanceos en las relaciones entre estados nacionales, subnacionales y 
municipales y modificaciones importantes en los montos de autonomía escolar en muchos países de la región. Por otra parte, se constató una mayor diversidad y utilización de instrumentos de regulación sobre el contenido que actuaban como "marcos de referencia" en combinación con el rediseño de planes y programas a nivel nacional y subnacional (DUSSEL, 2006): parámetros o contenidos mínimos nacionales, mallas curriculares, estándares y reglas de composición curricular.

Argentina, que sostuvo históricamente un principio federal "débil" en educación, debido al papel protagónico del Estado Nacional, produjo importantes traspasos de responsabilidad y gobierno a los estados provinciales. Esto, que se verá luego con mayor detalle, marca un movimiento hacia mayor distribución de autoridades y menor influencia de la órbita nacional. Brasil, por su parte, mostró algunos procesos de dirección inversa. En el marco de su constitución federativa mantuvo tres "sistemas" ligados con los niveles de gobierno: federal, estadual y municipal mediante un régimen de autonomía, interdependencia y colaboración (BONAMINO; MARTÍNEZ, 2002), previsto en la Constitución Federal y en la Ley de Directrices y Bases de la Educación Nacional de 1996. La nación cumple roles de coordinación, establecimiento de parámetros mínimos de calidad educativa y asistencia técnica y financiera. Los estados y los municipios están a cargo, en una relación de complementariedad y mediante distintos formatos, para atender la Enseñanza Fundamental de 6 a los 14 años de edad -que agrupa educación primaria y secundaria básica-. Mayoritariamente, la educación secundaria alta, que corresponde a los tres últimos años (15 hasta 17 años), está a cargo de los estados. De ellos depende el currículum según las Directrices Curriculares para la Enseñanza Media del año 2012. En el último período se han verificado políticas destinadas a redistribuciones de recursos y modos de coordinación sobre la base de la estructura previamente descentralizada. El Plan Nacional de Educación es el último documento elaborado por el Ministerio de Educación nacional que sirve de base para la planificación en educación para la nación, estados y municipios para el período 2014 a 2024; allí se propone construir formas orgánicas de colaboración entre los sistemas de enseñanza a partir de la definición de metas que articulen los distintos sistemas de con foco en los problemas de la calidad y la equidad en educación.

Otros países de gobierno central realizaron modificaciones radicales que tendían a una amplia autonomía por parte de las escuelas en la toma de decisiones sobre el currículum, como en Colombia (VÉLEZ, 2012) y en Chile (COX, 2006; VEZUB, 2014). 
Colombia llevó adelante una acentuada descentralización educativa y curricular a partir de la Ley General de Educación de 1993, que otorgó autonomía a las escuelas en cuanto a la definición del currículo y la realización de sus planes de estudio, en el marco de los lineamientos curriculares nacionales y los indicadores de logro fijados por el Ministerio de Educación Nacional. De allí que el nivel nacional cumpla un papel orientativo y sean las Secretarías de Educación de cada departamento que las que supervisen y asesoren el diseño y desarrollo del currículum en las escuelas. El decreto $\mathrm{n}^{\circ} 1290$ de 2009, reafirmó la autonomía de los establecimientos para definir el currículum y los criterios de promoción. En Chile, la sanción de la Ley Orgánica Constitucional de Educación de 1990 “[...] pone fin al monopolio ministerial sobre el currículum escolar, consagrando la autonomía de los establecimientos para producir sus propios programas de estudio, y creando un nuevo organismo para su control nacional, el Consejo Superior de Educación" (COX, 2006, p. 2) Por su parte, El Ministerio de Educación de Chile a través de su Unidad de Currículum y Evaluación diseñaba los lineamientos curriculares nacionales, objetivos fundamentales y contenidos mínimos ${ }^{3}$, que eran aprobados por el Consejo Superior de Educación y daba seguimiento sobre el cumplimiento de sus objetivos. Sin embargo, con el tiempo se acumularon evidencias sobre las desiguales posibilidades de las escuelas de enfrentar los problemas de la planificación curricular, por lo que, en 2009, la Ley General de Educación estableció como tarea del Ministerio Nacional la elaboración de planes y programas para el nivel primario y secundario de uso obligatorio en escuelas que no pudieran desarrollarlos en razón de su situación y/o recursos. Incluso Uruguay, que mantuvo un esquema centralizado de gobierno educativo - las decisiones sobre el currículum son tomadas por la Administración Nacional de Educación Pública mediante los Consejos Desconcentrados de Educación Primaria, Educación Secundaria y Educación Técnica- realizó una importante apertura a la planificación curricular en los centros escolares a partir del nuevo "Programa para la Educación Inicial y Primaria" del año 2008.

Como parte del panorama de innovaciones ocurridas en la región se debe tener en cuenta el amplio desarrollo, en un grado desconocido hasta el momento, de la evaluación del aprendizaje y de los distintos componentes del sistema. Como señala Vélez (2012) para el caso de Colombia "El sector pasó de medir muy pocas cosas a medirlo todo". La instalación y expansión de los sistemas nacionales de evaluación fue rápida y generalizada en el período. Esto impactó de manera diferencial en los países de la región, pero los sistemas de 
evaluación, a nivel nacional o estadual, generaron efectos sustantivos sobre la regulación del contenido y de la enseñanza. Por ejemplo, Colombia tiene evaluaciones externas nacionales -Pruebas Saber y de Estado-, Argentina instaló el ONE (Operativo nacional de Evaluación, Brasil cuenta con la Prova Brasil e importantes sistemas de evaluación estaduales y Chile desarrolló el Sistema de Medición de la Calidad Educativa (SIMCE) cuyos resultados "[...] si bien no determinan directamente el currículum prescripto, tienen mucha influencia en la asignación de recursos y la elección de escuelas por parte de los padres, con lo cual constituyen poderosas herramientas de regulación de qué se enseña y cómo se enseña en las escuelas." (DUSSEL, 2006, p. 18). Estos sistemas se caracterizaron, según Larripa (2003), por el principio de rendición de cuentas, como en los países desarrollados, y con la inversión en educación. Forman parte, además, de un marco de políticas tendientes a la descentralización de los modos de gobierno y gestión. También, su implantación quedó fuertemente ligada con la idea de "calidad" ya que, de un modo reductivo, se utilizó el rendimiento académico de los alumnos como una medida del funcionamiento de los demás componentes del sistema (TIANA FERRER, 1998).

En los últimos 10 años, a los fines declarados por los operativos nacionales de evaluación de la calidad de la educación, se le sumaron en la región las pruebas internacionales que pretenden proveer una visión comparativa para saber dónde están los diferentes países, respecto a un amplio espectro mundial, y adquirir visión y elementos interpretativos claves para definir políticas, tanto a nivel macro como en el quehacer de las unidades educativas (COX, 2004). De estos estudios, los que han tenido más impacto en la Región son el estudio PISA de la OECD y el TIMSS. Se ha desarrollado abundante debate acerca de la información que arrojan esas pruebas y del uso de medidas cuya comparabilidad es materia de discusión (LARRIPA, 2003; COSSE, 2006; TENTI FANFANI, 2003; RAVELA, 2006). En este marco, es necesario destacar pruebas regionales, como el SERCE y el TERCE, coordinados por UNESCO, que plantearon formas cooperativas para garantizar la validez de contenido de las pruebas y establecer parámetros más acordes con los sistemas nacionales participantes.

Sin ánimo exhaustivo puede afirmarse que en distintos países de la región se conjugaron distintas formas de autoridad e instrumentos para la regulación del currículum. Muchos de esos cambios obedecieron a la necesidad de lograr una mejor articulación entre los diversos tramos de la educación básica y obligatoria extendida y actualizar los contenidos de la transmisión escolar. Distintas alternativas se han desarrollado para atender estos requerimientos 
que se corresponden con tradiciones de gobierno, pero asimismo, con modificaciones de los patrones históricos para ordenar el sistema educativo. Ello ha generado, como se señaló a nivel internacional, movimientos en diversos sentidos. Lo cierto es que fueron comunes, aunque no generalizados, cambios en las distribuciones de autoridad, con más o menos intensidad, éxito y continuidad. El caso de Argentina muestra un proceso que, a lo largo de los últimos 25 años, combinó una gran parte de las variables, procesos, mecanismos e instrumentos puestos en juego. A ello se dedicará el próximo apartado.

\section{NUEVAS FORMAS DE GOBIERNO E INSTRUMENTOS CURRICULARES: EL CASO ARGENTINO}

\section{Hacia una mayor descentralización del gobierno educativo}

Históricamente, Argentina mantuvo un gobierno dual de la educación ya que, si bien la Constitución Nacional de 1853/1860 ponía en mano de los estados provinciales la educación primaria, sucesivas intervenciones del Estado Nacional condujeron a la creación de un extendido sistema de educación básica de dependencia nacional en coexistencia con los sistemas provinciales en los distintos territorios ${ }^{5}$. En la educación secundaria también se generaron sistemas bajo las dos dependencias. En ese contexto, las agencias nacionales (Consejo Nacional de Educación, Ministerio de Educación) jugaron un rol de enorme importancia en la dirección, gestión, financiamiento y orientación del conjunto del sistema. Sus normativas, Planes y Programas, fueron una referencia esencial para el resto de las unidades estatales (PALAMIDESSI, FELDMAN, 2003). En síntesis, no obstante las disposiciones constitucionales que consagraban el federalismo político, el gobierno efectivo del sistema educativo mantuvo una fuerte impronta centralizada.

En la década de 1990 se produjo un cambio profundo en la estructura del sistema escolar mediante leyes que completaron el traspaso de todos los servicios educativos (inicial, primaria, secundaria y superior), menos las universidades ${ }^{6}$, a las jurisdicciones provinciales y establecieron cambios en la estructuración de los niveles, la cantidad de años de obligatoriedad, el financiamiento educativo, y la delimitación de tareas entre la nación y las jurisdicciones provinciales ${ }^{7}$.

La Ley Federal de Educación introdujo el primer cambio radical en la estructura histórica del sistema educativo de dos tramos: primaria y secundaria. Crea la Educación General Básica obligatoria de 10 años ${ }^{8}$ dividida en tres ciclos (EGB 1, 2 y 3), seguida por la Educación Polimodal de 3 o 4 años (equivalente a una secundaria superior). El tercer Ciclo 
de Educación General Básica (EGB3) - que incluía el último año de la ex primaria y los primeros dos de la ex secundaria- tendía a funcionar, de hecho, como un nivel intermedio de escolarización bisagra entre la educación básica y la media superior. La falta de definición acerca de la estructura organizacional para la EGB3 (GALARZA, 2000) dejó un espacio abierto que dificultó extraordinariamente las tareas de coordinación, en un marco de multiplicidad de alternativas de los estados provinciales para resolver ese segmento de la escolaridad.

Como consecuencia de la transferencia de autoridad sobre el sistema y de la reforma de su estructura surgieron nuevas necesidades de coordinación, delimitación y creación de un marco unificado -en acuerdo con la tradición imperante en el país- que permitiera articular las formas históricas de gestión con los nuevos requerimientos de la reforma. Por un lado, la reforma del sistema obligaba a una redefinición de contenidos y funciones de los niveles. Por otra parte, exigía nuevos ámbitos de decisión sobre el currículum. De acuerdo con la normativa, se encomendó al Ministerio de Cultura y Educación nacional establecer, en acuerdo con los estados provinciales, los Contenidos Básicos y Comunes (CBC) para que las provincias elaboraran sus diseños curriculares y las instituciones sus proyectos educativos ${ }^{9}$. El Estado Nacional se reservó la facultad de evaluar los aprendizajes de los estudiantes a través de la implementación de los Operativos Nacionales de Evaluación de la Calidad (ONE). También se renovaron las relaciones de autoridad entre el estado nacional y las provincias a partir de la revitalización del Consejo Federal de Educación (CFE) como representación de la federación. El Consejo Federal de Educación era un organismo de planificación y coordinación entre las provincias, creado en los años setenta. Sus miembros eran los ministros de educación de las 24 jurisdicciones del país y sus disposiciones tenían valor sólo dentro de la voluntad política de las partes acordantes. La novedad es que en los años noventa, ante la transferencia total del sistema nacional, fue notoria la falta de un organismo de toma de decisiones federales. Se recurrió, entonces, a las resoluciones del CFE y se pretendió darles un status legal del cual, probablemente, carecían. De allí que fue problemático el carácter vinculante de todas las resoluciones adoptadas en el marco de la llamada "Transformación Educativa" de los años noventa -incluyendo los Contenidos Básicos Comunes-. Esta ambigüedad legal no resultó muy visible por la avasalladora fuerza política de la acción reformadora, la subordinación de los gobiernos provinciales a los mandatos del gobierno nacional y la ampliación de los aportes financieros por parte de los organismos nacionales lo que facilitó y potenció la iniciativa política. 
Con posterioridad a la profunda crisis política y social del año 2001, una nueva ley restituyó el sistema a su formato anterior (Educación Inicial, Primaria y Secundaria) y dispuso la obligatoriedad del nivel secundario completo (13/14 años de educación obligatoria, según la modalidad) ${ }^{10}$. También, de acuerdo con esta norma, las resoluciones del CFE adquirieren carácter vinculante y son de cumplimiento obligatorio por parte de los estados provinciales. Bajo este nuevo marco corresponde a los gobiernos provinciales definir y aprobar sus propios diseños curriculares en el marco de una pauta general acordada que define "[...] estructuras y contenidos curriculares comunes y núcleos de aprendizaje prioritarios en todos los niveles y años de la escolaridad obligatoria." "11 A partir de este segundo momento, el lugar central que ocupó la política curricular del Ministerio Nacional de Educación ${ }^{12}$ parece diluirse en relación a la posición estratégica que ocupó en los '90. Por un lado, porque la referencia que constituyen los Núcleos de Aprendizaje Prioritario es menor en comparación con su antecedente, los $\mathrm{CBC}$, ya que su intención es menos prescriptiva ${ }^{13}$. Por otro, porque en el transcurso de una década varias provincias desarrollaron mejores condiciones a través del fortalecimiento de sus equipos técnicos para definir su currículum. De hecho, las iniciativas políticas de muchas provincias tomaron distintas direcciones. En algunos casos, basados en dinámicas propias y muy ambiciosos en cuanto a su extensión y alcance: Buenos Aires (2007), Córdoba (2009) y Entre Ríos (2011); otras que han optado por adaptar más fuertemente las indicaciones de los NAP (La Pampa, Salta) y un grupo que sigue trabajando con documentos preexistentes a las disposiciones curriculares actuales, mayormente con los lineamientos de los Contenidos Básicos Comunes, (Santa Fe, San Luis) ${ }^{14}$.

\section{Nuevos instrumentos de gobierno del currículum}

Una novedad de las políticas curriculares es el uso de marcos curriculares de referencia, constitutivos de los procesos de descentralización educativa que se dieron en muchos países de la región (FERRER, 2004). En el caso argentino estos marcos de referencia cumplieron dos roles: algunos especificaban las bases para definir el contenido de enseñanza y sus formatos de organización y otros establecieron reglas para la composición del currículum por parte de las jurisdicciones y las escuelas.

En relación con la definición del contenido, como ya se vio, el proceso de reforma de los noventa incluyó, con posterioridad a la transferencia del sistema escolar a las provincias, la definición de los Contenidos Básicos Comunes (CBC) con el argumento de otorgar coherencia, integración y posibilidad de reconocimiento de estudios a nivel nacional a un sistema educativo que había desplazado, legalmente, 
la capacidad de decisión a las entidades estatales federadas. Los CBC fijaron las bases de contenidos para el diseño curricular de la Educación General Básica obligatoria y garantizaban la equivalencia educativa en cualquier sistema provincial. Para su elaboración se recurrió a comisiones de expertos académicos que se dedicaron a áreas disciplinares específicas y adoptaron una forma de organización de los contenidos por capítulos correspondientes a disciplinas escolares. En el marco de esta tradición disciplinar se consideró que tanto la selección del contenido, como los principios que rigen su organización y secuencia, debían basarse en la estructura de las disciplinas, con la intención de que las escuelas proporcionaran un conocimiento de valor académico y científico. Según los argumentos oficiales esgrimidos en favor de este procedimiento, este criterio permitiría, además, garantizar la enseñanza de los conceptos centrales de los respectivos campos disciplinares, ofrecer una presentación del contenido estructurada desde el punto de vista lógico y minimizar el riesgo de la falta de actualización del contenido (AMANTEA et al., 2006). La preocupación sobre los contenidos de corte disciplinar científico en la enseñanza, especialmente en las escuelas secundarias, había sido instalado, bajo la hipótesis de "la escuela vacía", por los trabajos realizados por un grupo de investigadores desde la FLACSO Argentina y ampliamente difundidos y aceptados en su momento por los medios académicos y el campo pedagógico ${ }^{15}$. Miembros de este grupo impulsarían, luego, la política de reforma del currículum de los años '90 desde el Programa de Asistencia Técnica para la Transformación Curricular en el Ministerio Nacional de Educación.

Acordados en el Consejo Federal de Educación, los CBC adquirieron un considerable status normativo, pese a que no eran prescriptivos desde el punto de vista legal ${ }^{16}$, y en la práctica definieron la matriz básica para la elaboración de los diseños curriculares de la mayoría de las provincias. Aunque nunca se realizó su evaluación sistemática, algunos análisis mostraron que se caracterizaban por un grado de exhaustividad que no se correspondía con la idea de niveles básicos (AMANTEA et al., 2006). También, el nivel de complejidad que proponían ponía en cuestión su capacidad de actuar como un efectivo marco para la programación curricular y la planificación de la tarea escolar. Puede decirse que, más que cumplir un papel de coordinación y unificación, los CBC fueron un ambicioso proyecto de modificación del contenido escolar según estándares de excelencia académica. Los supuestos que guiaron su elaboración merecen, todavía, un análisis 
exhaustivo ${ }^{17}$ pero lo relevante, es constatar las dificultades que se enfrentaron para fijar el mínimo unificador de contenidos y crear un instrumento adecuado desde el punto de vista de la evaluación.

La autoridades educativas que asumen con posterioridad a la aguda crisis económica y social de 2001/2002 encuentran una declinación evidente del impulso reformista, un creciente descontento con los resultados manifiestos de la reforma y una gran preocupación por el costo y esfuerzo que implicaba su completamiento en caso de seguir adelante. La principal preocupación residía en la fragmentación del sistema en la franja adicionada para cubrir los diez años de escolaridad obligatoria, correspondiente al EGB3. Los propósitos principales consistieron en crear pautas de ordenamiento de la situación, incluyendo la definición de contenidos para los niveles escolares. Uno de los proyectos consistió en reelaborar un marco de contenidos tratando, esta vez sí, de atender al carácter mínimo y básico de la prescripción: los Núcleos de Aprendizajes Prioritarios (NAP) que, sin embargo, no derogaron los CBC y mantuvieron una doble, y poco clara, legalidad. El pasaje de los CBC a los NAP's muestra un cambio en los principios utilizados para la definición de contenidos mediante un tránsito desde formas centradas en temas hacia un planteo de situaciones didácticas (FEENEY, 2006). Aún así, el rasgo compartido por ambos dispositivos curriculares es la primacía de la organización en base a contenidos disciplinares, algo que distingue a Argentina de otros países de la región que adoptaron el enfoque por competencias.

La definición de los NAP descansó, una vez más, en un dispositivo de especialistas. Esta vez sin el elevado nivel científico o académico de los convocados durante 1995 y 1996, pero que compartían un principio básico de jerarquía disciplinar, agregando una tradición poco influyente en el Ministerio en la década anterior: la de la especialización didáctica ${ }^{18}$. Estos especialistas continuaron adheridos a la lógica de autonomía y jerarquía disciplinar que formó parte del espíritu de los noventa. Solo que en esta oportunidad su trabajo no mereció especial atención por parte de los tomadores de decisiones.

Esta tendencia dejó, por tanto, el campo abierto a los especialistas disciplinares quienes, carentes de orientación, dieron a la decisión política general un sesgo propio que respondió, básicamente, a un entramado autosostenido de grupos profesionales motivados por una ideología propia de renovación escolar (FELDMAN, 2006, p. 92) ${ }^{19}$.

Los esfuerzos por ordenar los mínimos de contenidos fueron acompañados en los noventa por la creación de marcos para la composición del currículum a cargo de los estados provinciales. 
Durante ese período, el Ministerio de Educación de la Nación impulsó en el Consejo Federal de Educación acuerdos para establecer los criterios de diseño curricular de la Educación General Básica (A16) y de la Educación Polimodal (A17). Ambos documentos definen una matriz que permite organizar y distribuir en el tiempo los contenidos a enseñar en el tramo correspondiente del sistema educativo, de acuerdo con criterios y parámetros que funcionan como reglas del juego para la elaboración de los diseños curriculares jurisdiccionales ${ }^{20}$. El impacto que tuvieron estos acuerdos federales en la elaboración de los diseños curriculares jurisdiccionales fue altísimo. El análisis de los textos provinciales muestra una importante homogeneidad en relación a los productos finales que avanzaron en la producción de los diseños muy ajustados a los marcos regulatorios. Terigi (2007) señala que en la concepción sobre el planeamiento educativo que imperó en los noventa se produjo una división del trabajo de planificación y ejecución entre nación y provincias. Ya fue señalado que el impulso reformista de los años noventa fue perdiendo vigor con el inicio de una profunda crisis económica y social y estaba prácticamente detenido antes del final del gobierno de Carlos Menem, bajo cuyo mandato se había iniciado. Luego del estallido de la crisis del 2001/2002 y el inicio de la recomposición económica, política y social a partir de año 2003, las nuevas políticas emprendidas pusieron en cuestión esa concepción de planeamiento. Los nuevos Planes de Estudio requeridos para adaptar los lineamientos curriculares a la recompuesta estructura del sistema se elaboran en base a marcos aprobados por el Consejo Federal de Educación ${ }^{21}$ que establecía parámetros mínimos e implicaban nivel mayor de generalidad y de menor de prescripción que las normativas anteriores. Además, cobraron mayor autonomía los equipos de especialistas de los distintos ministerios de educación de las jurisdicciones, lo que disminuyó en alto grado la influencia de las autoridades nacionales, y sus equipos técnicos, en el rediseño de los Planes de Estudio.

Ya fue planteado que, estrechamente ligado con la fijación de marcos curriculares de referencia, se encuentra el énfasis en los procesos y sistemas de evaluación externos a la escuela. En Argentina, este papel lo cumplieron los Operativos Nacionales de Evaluación (ONE) -el instrumento creado para medir el rendimiento de los estudiantes en las áreas básicas del currículum- administrados por el Ministerio de Educación nacional. Los contenidos a evaluar son acordados entre la Nación y las provincias sobre la base de los Diseños Curriculares Jurisdiccionales, los NAP, los resultados de los 
ONE anteriores y los libros de textos más utilizados en el país. Sin embargo, la discontinuidad del funcionamiento del Sistema Nacional de Evaluación de la Calidad operó como obstáculo importante a la hora de tomar decisiones sobre la marcha de los procesos de cambio del currículo y la mejora de las prácticas docentes. Además, la difusión de los resultados ha sido bastante discrecional y ha faltado claridad en su uso por parte de las autoridades. De allí que, a diferencia de otros países de la región, en Argentina se ha hecho un uso muy limitado de la evaluación para dirigir políticas de mejora y no se ha instalado la propia idea de evaluación como un requisito para el funcionamiento y gestión de los distintos subsistemas. Marca, en ese sentido, un rasgo diferencial cuyas consecuencias requieren, aún, una profunda revisión.

\section{COMENTARIO FINAL}

En el caso argentino pueden verse puestos en juego muchos de los "ingredientes" que están ligados a las olas de reforma educativa de los últimos 30 años, en especial la profundización del movimiento de descentralización educativa, la definición de leyes generales que regulan la extensión de la obligatoriedad, pasando por los vaivenes en el ordenamiento (y posterior reordenamiento) de la estructura de los niveles y ciclos educativos, la definición de marcos de referencia sobre los contenidos concebidos con valor social para ser enseñados en las escuelas y de criterios generales de composición de la oferta formativa. Se suma, también, la implementación de sistemas de evaluaciones externos a la escuela que, si bien tuvieron cierto protagonismo en el inicio de los años ' 90 , fueron perdiendo presencia en la comunidad educativa ni bien iniciado los años 2000. A diferencia de otros países de la región, cabe destacar el menor énfasis que en las políticas nacionales se concedió a la autonomía escolar.

Las experiencias reseñadas en el trabajo mostraron distintas "situaciones" frente a la regulación sobre el currículum. El caso de Argentina, en particular, permite ver que las diferentes dimensiones atendidas por sus acciones sobre el currículum en estas décadas tuvieron como origen lógicas de encadenamiento de reformas de varios niveles. La finalización de la transferencia completa de todo el sistema educativo no universitario a las provincias, a inicios de la década de 1990, formó parte de reformas más generales dirigidas a reconfigurar el papel del Estado Nacional. Esto se combinó con la reestructuración del sistema de niveles y ciclos escolares y una 
ampliación de la obligatoriedad escolar. Allí residió el origen de una acelerada, compleja, y por momentos contradictoria, tarea para mantener coordinación nacional en un país que históricamente se movió entre dos lógicas, mantuvo un sistema dual, de dependencia provincial y nacional, y se caracterizó por un predominio de las normativas y directivas nacionales para la regulación del currículum. Las acciones curriculares fueron, principalmente, parte de reformas de estructura del sistema y de las formas de gobierno y financiamiento educativo. De allí que, en ese caso, las políticas hacia el currículum hayan tenido una fuerte motivación política del impulso generado por otros movimientos. Este impulso, avasallador en su momento, se articuló con líneas de pensamiento académico acerca de recuperar la capacidad escolar de transmisión de "conocimientos socialmente válidos", con un extendido consenso acerca de la necesidad de contar con una ley integral de educación - que el país nunca había tenido- y con históricos proyectos de creación de una escuela intermedia. En el marco de un proceso de reestructuración del papel del Estado, los portadores de estos puntos de vista encontraron la oportunidad legislativa y lugares en puestos relevantes del Ministerio Nacional para promover un intenso y acelerado trabajo sobre el currículum cuya trayectoria todavía requiere evaluación. También puede verse como estos cambios en la estructura del sistema instalaron novedades en el gobierno del contenido escolar y, en un marco en el que se sucedieron movimientos a favor de la descentralización y vaivenes hacia el centro en un juego de equilibrios inestables, se gestaron nuevos instrumentos de control del currículum.

Es innegable que, en el período considerado, Argentina asistió a potentes acciones en relación con las políticas curriculares. Los movimientos que se sucedieron atravesaron y fueron atravesados por intensas -y por momentos dramáticas- vicisitudes políticas y sociales y mostraron un encadenamiento de acciones que respondió a un flujo variable de fuerzas y condiciones en el cual se produjeron respuestas a los problemas que abría el avance progresivo de la reforma en los noventa y a su posterior revisión y acomodamiento en la década siguiente. Los análisis futuros de este proceso, como de anteriores, deberían probablemente mantener ciertas precauciones frente al impulso a interpretar lo realizado solo como un proceso con objetivos preplanificados o definidos desde estrategias macropolíticas de largo alcance. Tal vez sea adecuado interpretarlos mediante algún tipo de análisis que, de manera ya clásica, Lindblom (1992) denominaba 
"incremental" - según el cual buena parte de la decisión política se entiende como "una ciencia de salir del paso"- o que Elmore y Sykes (1991, p. 190) definían como un flujo de problemas y soluciones que convergen, frecuentemente de maneras aleatorias, en torno a eventos críticos. Es probable que Argentina atraviese una larga e incierta etapa de transición en sus formas de gobierno sobre el currículum y el contenido escolar. Esta etapa, caracterizada por el debilitamiento del histórico peso de los poderes centrales y de las vías administrativas para la gestión del currículum, no ha encontrado nuevas formas de equilibrio entre los organismos nacionales y juridiccionales, nuevas formas de relación entre las agencias de gobierno educativo y las unidades del sistema escolar y nuevas maneras y estrategias efectivas de intervención sobre el contenido escolar, su transmisión y apropiación/aprendizaje.

Aunque impactaron principalmente en la escuela secundaria, los cambios introducidos condujeron a redefinir el papel del conjunto de los niveles y pusieron en tensión las tradiciones relativas al currículum y la definición de los mínimos básicos para cada momento del trayecto educativo. Uno de los principales problemas que se plantea a las políticas curriculares es el de acompañar la expansión de la cobertura y de los años de escolaridad promedio que se produjo en toda la región, con mejoras en la calidad de la enseñanza y de los aprendizajes. Este ya es, seguramente, un punto de no retorno a partir del cual las siguientes iniciativas de reforma curricular deberán buscar formas de compatibilizar las exigencias asociadas con la incorporación de sectores excluidos o precariamente incluidos en los sistemas educativos, con la búsqueda de mayores niveles de calidad, pertinencia y actualización de los conocimientos, valores y competencias a desarrollar en los estudiantes. Una cuestión que se liga de manera especial con el currículum y no solo con factores externos -como las condiciones sociales y culturales de partida de los estudiantes- o internos -como la pedagogía, la evaluación o la organización escolar.

Debe tenerse en cuenta que en el recorrido realizado se consideraron movimientos amplios para combinar mayor autonomía escolar y local con diversos grados de coordinación nacional. También que trataron de responder al desafío de garantizar cierta equivalencia en el mínimo de educación imprescindible y común en formatos que, por lo general, renunciaron al control centralizado de tipo administrativo. Estos movimientos muestran distintos recorridos tomando en cuenta los puntos de partida originales de cada sistema. Todo esto dicho desde un punto de vista limitado como fue el utilizado en este trabajo 
al proponer ejemplos de cambios en las distribuciones de autoridad sobre el currículum. Por supuesto que una ponderación más profunda de estos recorridos y movimientos no puede soslayar una cuestión que, aunque aquí no fue expuesta, debe ser siempre tenida presente: las bases políticas y económicas que sustentaron estos movimientos y políticas fueron diferentes y respondieron a distintas motivaciones relativas a cambiantes redistribuciones de poder, proyectos económicos y redefiniciones del papel del estado en la región durante este período.

Por último, puede señalarse que la revisión del proceso argentino, en el marco de las tendencias que muestran otros países de sudamérica, muestra rasgos que pueden expresar un giro de similar importancia al que provocó en la décadas del 60 y 70 la llegada de la teoría curricular de origen anglosajón. Las formas tradicionales de regular el currículum, que pueden caracterizarse como "modelo burocrático de gestión", funcionaron con eficacia desde la constitución de los sistemas educativos hasta bien avanzado el siglo XX. Estados centrales fuertes, consenso social sobre el valor de la educación, expansión exitosa del sistema y una sólida alianza entre sectores intelectuales, gobierno del sistema educativo y actividad escolar, garantizaron un buen funcionamiento durante el período de gestión administrativa en manos del Estado que se extiende hasta fines de los sesenta. Pero a partir de ese momento se producen influencias significativas que afectan a los mecanismos de gestión. Junto a los procesos de modernización impulsados por iniciativas continentales como la Alianza para el Progreso y la acción de organismos como la Organización de los Estados Americanos o la UNESCO, se promueve la formación de recursos técnicos, la planificación curricular mediante la formulación y taxonomías de objetivos operacionales y la teoría de la evaluación. También se pone en circulación ideas en torno a la descentralización y desconcentración de los sistemas educativos (PALAMIDESSI, FELDMAN, 2003). Hacia los noventa, los intentos de elaborar e implementar políticas sobre el currículum transitan caminos muy distintos a los tradicionales. Los dispositivos de control tienden a distribuirse y diversificarse, los intereses en política educativa se vuelven más permeables a lógicas locales, las burocracias tradicionales pierden capacidad de regulación, surgen nuevas capas técnicas, se desarrollan innovaciones en las formas de gobierno educativo, se flexibilizan y articulan las formas de financiamiento relacionadas con la mejora, se asiste a generalizados procesos de descentralización y distribución de la toma de decisiones 
y se crean instrumentos para articular la planificación curricular en los distintos niveles estatales. En resumen, junto a un cambio de grandes proporciones en el currículum de la región, se desarrolla un nuevo entramado de dispositivos técnicos que altera de manera significativa las formas de gobierno sobre el currículum. Es probable que el conjunto de procesos reseñados hasta aquí visibilicen al currículum como un problema de política y, especialmente, como política pública, algo que marcó una dimensión importante en las reformas educativas de los últimos veinticinco años en América del Sur.

\section{REFERENCIAS}

AGUERRONDO, I.; NÚÑEZ PRIETO, I.; WEINSTEIN CAYUELA, J. Institucionalidad de los ministerios de Educación: Los procesos de reforma educativa de Argentina y Chile en los años noventa. IIPE- UNESCO, 2010. <http://www.iiep.unesco.org/fileadmin/user_ upload/Cap_Dev_Rethinking/pdf/CapDev_Spanish.pdf>. Consulta: ago. 2011.

AMANTEA, A. et al. Propostas curriculares na Argentina: as tradiçoes disciplinar, da didática peral e das didáticas especiais. En: CASIMIRO LOPES, A.; MACEDO, E. (Org.). Políticas de currículo em múltiplos contextos. Sao Paulo: Cortez Editora, 2006. (ISBN 85-249-1225-1). p. 38-69.

AKKER, J. Curriculum perspectives: an introduction. En: VAN DEN AKKER, J.; KUIPER, W.; HAMEYER, U. (eds.): Curriculum landscape and trends. Dordrecht: Kluwer Academic Pres, 2004.

BANCO MUNDIAL Ampliar oportunidades y construir competencias para los jóvenes: una nueva agenda para la educación secundaria. Bogotá: Banco Mundial-Mayol Ediciones, 2007.

BELLEI CARVACHO, C. Políticas educativas para el nivel secundario: complejidades y Convergencias. En: TENTI FANFANI, E. La escolarización de los adolescentes. Buenos Aires, IIPE UNESCO, 2012. p. 215-216.

BONAMINO, A.; MARTÍNEZ, S. Directrizes e parámetros curriculares nacionais para o ensino fundamental: a participacao das instancias políticas do estado. Revista Educacao e sociedade, Campinas, v. 23, n. 80, p. 368-385, sept. 2002.

CERVINI, R.; TENTI FANFANI, E. Notas sobre la masificación de la escolarización en seis países de América Latina. Buenos Aires: SITEAL, OEI - IIPE/UNESCO, 2007.

COX, C. Poder, conocimiento y sistemas educacionales: un modelo de análisis y cinco proposiciones para un programa de investigación sobre transmisión cultural escolar en Chile. Educaçao e Realidade, Porto Alegre, v. 14, n. 1, p. 55-69, jan./jun.1998.

COX, C. Reformas curriculares y evaluación internacional de resultados de aprendizaje categorías analíticas y datos para la reflexión. Material de apoyo para actividades del Seminario de discusión de la problemática educativa actual para cuadros superiores de Ministerios de Educación de las Provincias, organizado por Ministerio de Educación, Ciencia y Tecnología de Argentina y el IIPE, 2004.

COX, C. Construcción política de reformas curriculares: el caso de chile en los noventa. Revista de Currículum y Formación del Profesorado, v. 10, n. 1, 2006. <http://www. ugr.es/local/recfpro/Rev101ART5.pdf>. Consultado: jun. 2015. 
COSSE, G. La evaluación internacional de la calidad educativa: estado de la cuestión, avances y dificultades. Ministerio de Educación, Ciencia y Tecnología. Unidad de Investigaciones Educativas. DINIECE. Argentina, 2006.

DE ALBA, A. Curriculum: crisis, mito y perspectivas. Buenos Aires: Miño y Dávila, 1995. DOLL, W. Currículo: uma perspectiva pós-moderna. Porto Alegre: Artes Médicas, 1997.

Dussel, I. Estudio sobre gestión y desarrollo curricular en países de América Latina. Ponencia presentada en el contexto de la Segunda Reunión del PRELAC, OREALC/UNESCO. Santiago de Chile, 2006. <http://www.elcorreo.eu.org/IMG/pdf/doc-1252.pdf>.

ELMORE, R.; SYKES, G. Curriculum Policy. En: JACKSON, P (ed.). Handbook of Research on Curriculum. New York: Toronto, Macmillan, 1992. p. 185-215.

FEENEY, S. La Política curricular en Argentina: ¿todo vale?. En: MONTERO, A. (Org.). Novas subjetividades, currículo, docência e questoes pedagógicas na perspectiva da inclusao social. Recife: Ediçoes Bagaço, 2006. (ISBN 85-373-0079-9). p. 341-354.

FELDMAN, D. El papel de la reforma curricular y de los expertos en la definición del contenido escolar. Revista Argentina de Educación, Buenos Aires v. 16, n. 25, p. 33-48, 1998.

FELDMAN, D. Notas sobre a política de curriculo na Argentina. Curriculo sem fronteiras [revista electrónica], v. 6, n 2. p. 82-97, jul./dez. 2006. (ISSN 1645-1384. 2006).

FELDMAN, D; ATORRESI, A; MEKLER, V. Planes y programas para mejorar el aprendizaje y reducir el fracaso en la Educación básica en América Latina. Revista Latinoamericana de Educación Comparada, Buenos Aires, n. 4, p. 12-24, 2013. (ISSN 1853-3744). <www. saece.org.ar/relec/numero4.php>.

FERRER, G. Las reformas curriculares de Perú, Colombia, Chile y Argentina: ¿quién responde por los resultados. Lima, Gupo de Análisis para el Desarrollo GRADE. Documento de trabajo n. 45, 2004. <http://bibliotecavirtual.clacso.org.ar/Peru/ grade20100511014531/ddt45.pdf>

FRIGERIO, G. et al. Curriculum presente, ciencia ausente. Normas, teorías y críticas. Buenos Aires: Miño y Dávila, 1991.

GAJARDO, M. Reformas Educativas en América Latina. Balance de una Década. Documento del PREAL Documentos, n. 15, 1999. <http://www.preal.org/BibliotecaN. asp?Pagina $=5 \& I d \_C a r p e t a=64 \&$ Camino=63 $\mid$ Pral Publicaciones $/ 64>$. Consulta: ago. 2011.

GALARZA, D. La estructura curricular del tercer ciclo de la EGB en ocho jurisdicciones. Ministerio de Educación de la Nación. Subsecretaría de Educación Básica. Unidades de Investigaciones Educativas (Informe de Investigación), 2000.

GOODSON, I. Historia del currículum: la construcción social de las disciplinas escolares. Barcelona: Pomares-Corredor 1998.

GOODSON, I. Procesos socio-históricos del cambio curricular. En: BENAVOT, A.; BRASLAVSKY, C. El conocimiento escolar en una perspectiva histórica y comparativa: Cambios de currículos en la educación primaria y secundaria. Buenos Aires: Granica, 2006. p. 335-350.

KAUFMAN, R.; NELSON, J. Políticas de Reforma Educativa: comparación entre Países. Documentos de PREAL, n. 33, 2005. < http://www.preal.org/BibliotecaN.asp?Pagina=3\&Id_ Carpeta $=64 \&$ Camino $=63 \mid$ PrealPublicaciones $/ 64 \mid$ PREAL Documentos $>$. Consulta: ago. 2011. KOGAN, M. Formas de gobierno y evaluación de los sistemas educativos. En: PEREYRA, M.; GARCÍA, J.; GÓMEZ, A.; BEAS, M. (comps.). Globalización y descentralización de los sistemas educativos. Barcelona: Ediciones Pomares Corredor, 1996. 
KRAWCZYK, N. R.; VIEIRA, V. L. Homogeneidad y heterogeneidad: un estudio comparativo sobre la reforma educativa de la década del 90 en Argentina, Brasil, Chile y México. Estudios Pedagógicos, Valdivia, v. 33, n. 2; p. 59-80, 2007. < http://www.scielo.cl/pdf/estped/v33n2/ art04.pdf $>$. Consulta: ago. 2011.

KUIPER, W.; AKKER, J. Curriculum development in (de)centralized policy contexts: Emerging dilemmas. Paper presented at the AERA 2005 Annual Meeting, Montreal, p. 1115, apr. 2005.

LARRIPA, S. El Sistema Nacional de Evaluación de la Calidad Educativa. (SINEC): acerca de la comparabilidad de sus resultados. Argentina 1996-2000. Tesis de Maestría. Universidad de San Andrés, 2003.

LINDBLOM, C. "La ciencia de salir del paso" y "Todavía tratando de salir del paso". En: AGUILAR VILLANUEVA, L. (ed.). La hechura de las políticas. México: Porrúa, 1992. p. 201- 254.

Ministerio de Educación de Brasil. Plan Nacional de Educación (PNE): Planejando a próxima década: conhecendo as 20 metas do plano Nacional de Educacaon (2014-2024). 2014.

ORNSTEIN, A.; BEHAR, L. (Ed.). Contemporary issues in curriculum. Boston: Allyn and Bacon, 1995.

PALAMIDESSI, M.; FELDMAN, D. The development of curriculum thinking in Argentina. En: PINAR, W. (ed.). Handbook of International Curriculum Research. New Jersey, Londres: Lawrence Erlbaum Publishers. 2003. p. 109-122. (ISBN 0-8058-3222-X, ISBN 0-8058-4535-6).

PALAMIDESSI, M. Desarrollos curriculares para la educación básica en el Cono Sur: prioridades de política y desafíos de la práctica. IBE Working Papers on Curriculum Issues, Ginebra, Suiza, n. 5, 2006. <http://www.ibe.unesco.org/sites/default/files/ resources/wpci-05-descurr_southamer_spa.pdf $>$.

PINAR, W. et al. Understanding Curriculum. New York: Peter Lang Publishing, 1995.

PINAR, W. Curriculum: toward new identities. New York: Garland Publishing Inc., 1998.

RAVELA, P. Para comprender las evaluaciones educativas. Fichas didácticas n. 1, 3, 4, 5 y 7. Santiago de Chile: Programa de Promoción de la Reforma Educativa en América Latina y el Caribe - PREAL, 2006.

SERCE.Los aprendizajes delos estudiantes de América Latinay elCaribe. Santiago de Chile: OREALC/UNESCO, 2008. <http://unesdoc.unesco.org/images/0016/001606/160660S. pdf>. Consulta: dic. 2010.

Tedesco, J. C.; Tenti Fanfani, E. La reforma educativa en la Argentina: semejanzas y particularidades. Buenos Aires, Proyecto "Alcance y resultados de las reformas educativas en Argentina, Chile y Uruguay", Ministerios de Educación de Argentina, Chile y Uruguay, Grupo Asesor de la Universidad de Stanford/BID, 2001. <http://info.worldbank.org/ etools/docs/library/109266/reforma\%20educativa\%20final.pdf>. Consulta: ago. 2011.

TEDESCO, J. C; LÓPEZ, N. Desafíos a la educación secundaria en América Latina. Revista de la Cepal, Santiago de Chile, n. 76, p. 66-69, 2002. <http://repositorio.cepal. org/bitstream/handle/11362/10801/076055069_es.pdf?sequence=1>. Consulta: jul. 2002 .

TENTIFANFANI, E. Los docentes y la evaluación. IIPE-UNESCO: Evaluar las evaluaciones. Una mirada política acerca de la evaluación de la calidad. Buenos Aires, Argentina, 2003.

TERIGI, F. Cuatro concepciones sobre el planeamiento educativo en la reforma educativa argentina de los noventa. Archivos Analíticos de Políticas Educativas, v. 15, n. 10, 2007. Recuperado de: <http://epaa.asu.edu/epaa/> Consulta: 19 jul. 2010.

TIANA FERRER, A. Tratamiento y uso de la información de evaluación. Buenos Aires, 
Ministerio de Cultura y Educación de la Nación-OEI. Serie de documentos del Programa de Evaluación de la Calidad de la Educación de la Cumbre Iberoamericana de Jefes de Estado y de Gobierno. v. 21998.

VAN DEN AKKER, J. “Currículum perspectives: an introduction”. In: VAN DEN AKKER, W. Kuiper; HAMEYER, U. (Ed). Currículum landscape and trends. Dordrecht. Kluwer Academic Publisher. 2004.

VELEZ, C. La gestión de la educación en Colombia 2002-2010. PREAL, Documento de trabajo n. 60, Santiago de Chile, 2012. (ISSN0718-6002).

VEZUB; L. (Coord.), VINACUR, T.; CARIDE, E. Estudio comparado de la calidad educativa en América Latina y el Caribe. Venezuela: UNICEF, 2014. < http://www.avec. org.ve/imagenes/pagina_web_2/noticias/noticia_102/seminario/3-5.pdf > .

\section{DOCUMENTOS}

ARGENTINA. Ley No 24195 Federal de Educación, 14 de abril de 1993. Boletín Oficial de la República Argentina. <https://www.boletinoficial.gob.ar/web2/utils/pdfView?file $=\% 2 \mathrm{Fpdf} \% 2 \mathrm{Fnormativa} \% 2 \mathrm{~F} 247861 \% 2 \mathrm{Fnull} \% 2 \mathrm{FGFoD} 1 \mathrm{rH} 4 \mathrm{tzEEqhS6V} 95 \mathrm{dFi} 1 \mathrm{bLS} 1 J V i 1$ Lcq5\%2BQdSUxI1Z9sUMkEIkJg\%3D>.

ARGENTINA. Consejo Federal de Educación. Acuerdos Federales para la implementación de la Ley Federal de Educación (1994). Serie 0, n. 1, n. 16, n. 17. <http://www.me.gov. ar/consejo/documentos/cf_documentos.html>. Ministerio de Cultura y Educación de la Nación-Consejo Federal de Cultura y Educación. Argentina.

ARGENTINA. Ley n² 26.206 Nacional de Educación 27 de diciembre de 2006. Boletín Oficial de la República Argentina. <https://www.boletinoficial.gob.ar/pdf/normativa /69833/null/GFoD1rH4tzEEqhS6V95dFi1bLS1JVi1bLcq5+QdSUxI1Z9sUMkEIkJg=>

ARGENTINA. Resolución no 225/04 C.F.C. Y E. Núcleos de Aprendizajes Prioritarios. Primer Ciclo EGB/nivel Primario. Ministerio de Educación, Ciencia y Tecnología. Buenos Aires, 11 de agosto de 2004. <http://www.me.gov.ar/curriform/nap.html>.

\section{NOTAS}

${ }^{1}$ La apertura del nivel se produjo mediante ramas técnicas o vocacionales dirigidas a facilitar el acceso de otro público y diferenciadas en el formato escolar y en el currículum. Estas estructuras -bipartitas o tripartitas- ampliaron las funciones exclusivamente preparatorias para estudios superiores de la escuela media mediante caminos escolares especializados y expresaron, como señala Ivor Goodson (1998), la capacidad del currículum para designar y, simultáneamente, para diferenciar.

${ }^{2}$ Ver Plan de Educación Nacional 2014-2024: "Planejando a próxima década: conhecendo as 20 metas do plano Nacional de Educação". Ministerio de Educación de la Nación, Brasil.

${ }^{3}$ Un criterio modificado a partir de la ley de 2009 momento a partir del cual se opta por la vía de fijación de resultados de aprendizaje expresados en Objetivos de aprendizaje.

${ }^{4}$ Cabe señalar que la instalación de sistemas de evaluación en Latinoamérica y el Caribe se lleva, principalmente, en el marco de préstamos del Banco Mundial y el Banco Interamericano de Desarrollo destinados a financiar las reformas educativas en curso. Como requisito de los planes y proyectos a financiar (destinados fundamentalmente a los niveles de enseñanza 
básica y media) las entidades multilaterales de crédito consideraron preciso que los países acreedores instalaran sistemas nacionales de evaluación de la educación, de forma tal de poder contar con información acerca de los resultados y de los réditos de la inversión realizada.

${ }^{5}$ En el marco de la Ley 1420 de Educación Común en 1884 - cuya vigencia se extendería por más de un siglo y establecía, para todo el país, la organización del sistema de educación primaria y sus contenidos mínimos- y la Ley Lainez de 1905 que autorizaba la creación de escuelas nacionales en territorios provinciales.

${ }^{6} \mathrm{La}$ transferencia de escuelas primarias fue realizada durante la dictadura militar hacia 1980, culminando sucesivos intentos de entregar ese sistema a las provincias que ya tenía antecedentes, aunque limitados, en décadas anteriores.

${ }^{7}$ Las Leyes de Transferencia de los Servicios Educativos N 24.049, 24.061 del año $1992 \mathrm{y}$ Decreto 964/99 y la Ley Federal de Educación - $\mathrm{N}^{\circ} 24.195$ del año 1993

${ }^{8}$ La Educación General Básica se extendía desde los 5 años -nivel Inicial-, abarcaba los siete de escuela primaria e incluía los dos primeros de la ex secundaria.

9 "Establecer en acuerdo con el Consejo Federal de Cultura y Educación, los objetivos y contenidos básicos comunes (CBC) de los currículos de los distintos niveles, ciclos y regímenes especiales de enseñanza -que faciliten la movilidad horizontal y vertical de los alumnos/asdejando abierto un espacio curricular suficiente para la inclusión de contenidos que respondan a los requerimientos provinciales, municipales, comunitarios y escolares". (Artículo 53, inc. B).

${ }^{10}$ Ley Nacional de Educación N ${ }^{\circ} 26.206$ del año 2006 que propuso reacomodar los elementos críticos resultantes de la aplicación de la Ley Federal

${ }^{11}$ (Ley Nacional de Educación, Art. 85).

${ }^{12}$ El término "Ministerio Nacional de Educación" se utiliza en sentido genérico ya que, en estos años el organismo cambió varias veces su denominación: Ministerio de Educación, de Educación y Cultura, de Educación, Ciencia y Tecnología-

${ }^{13}$ Como señala la Introducción (2004): "Por ello, dado que las distintas Provincias y la Ciudad de Buenos Aires cuentan con sus propios diseños curriculares, el abordaje de estos Núcleos en las escuelas se realizará según las formas particulares que en cada Jurisdicción se propongan";

${ }^{14} \mathrm{En}$ los todos los casos, el nivel referido es el nivel primario de educación.

${ }^{15}$ Los resultados de ese grupo de trabajo se pueden ver en Frigerio et al. (1991).

${ }^{16}$ De hecho, las provincias de Neuquén, Río Negro y la Ciudad Autónoma de Buenos Aires, que se mantuvieron apartadas del proceso de reforma de la década pasada)

${ }^{17}$ Una reflexión sobre este asunto fue avanzada en Feldman (1998).

${ }^{18}$ En el artículo mencionado Amantea, Cappelletti, Cols y Feeney (2006) describen tres tradiciones en las que se inscribe la teoría y la práctica curricular en Argentina: la disciplinar, la de las didácticas especiales y la de la didáctica general. Esta última, prácticamente ignorada en los procesos de reforma curricular de la última década.

${ }^{19}$ La traducción es de los autores.

${ }^{20}$ Estas incluyen: los capítulos de contenidos; la carga horaria mínima de los diferentes ciclos y de la jornada escolar; la cantidad de horas destinadas al tratamiento de los CBC y las fijadas por las provincias para distribuir entre tutorías, orientación y contenidos de definición institucional; el máximo de asignaturas por año y un sistema de distribución de asignaturas mediante alternativas por espacios disciplinares.

${ }^{21}$ Mediante la Resolución 84/09 para los aspectos generales y resoluciones específicas para las distintas orientaciones de la escuela secundaria. 
Recibido: $10 / 08 / 2015$

Aprobado: 18/02/2016

Correo postal:

Silvina Feeney

Juan María Gutierrez 1150 (entre José León Suarez y Verdi)

Los Polvorines - Pcia de Bs. As. Argentina

Conmutador: 4469-7500 - C.P. 1613

Daniel Feldman

Lavalleja 297, 4 A (CABA 1414) Argentina 